\title{
Artigo Original / Original Paper \\ Relações ecológicas entre orquídeas e forófitos em Floresta Ombrófila Mista sob influência nebular, Planalto Serrano Catarinense
}

Ecological relationships between orchids and phorophytes in Upper-Montane Araucaria Forest with cloud influence, Southern Plateau, Santa Catarina

Karina Montibeller-Silva ${ }^{1,2,6}$, Juliano Pereira Gomes ${ }^{1,3}$, Adelar Mantovani ${ }^{1,4}$

\& Roseli Lopes da Costa Bortoluzzi ${ }^{1,5}$

\begin{abstract}
Resumo
Este estudo objetivou conhecer a riqueza e estrutura de orquídeas epífitas e seus forófitos, avaliar as interações entre estas duas assembleias, bem como, amostrar as espécies forofíticas e não forofíticas para orquídeas em um remanescente de Floresta Ombrófila Mista sob influência nebular no Planalto Serrano Catarinense. A amostragem foi realizada no Complexo Serra da Farofa, em uma parcela de $5.000 \mathrm{~m}^{2}$. A riqueza e estrutura das assembleias arbóreas, forofítica e não forofítica e de orquídeas foram avaliadas. Três espécies de orquídeas foram registradas, colonizando 15 espécies de forófitos. A distribuição espacial dos forófitos e orquídeas foi agregada. Os forófitos colonizados por orquídeas apresentaram altura e Diâmetro a Altura do Peito (DAP), significativamente maiores que demais forófitos. O ritidoma do tipo rugoso foi o mais comum em forófitos colonizados por orquídeas. Drimys angustifolia (Winteraceae) e Myrceugenia euosma (Myrtaceae) foram as principais espécies forofíticas, cruciais para a manutenção da assembleia de orquídeas neste local. As informações deste manuscrito destacam a proteção in situ de Cattleya coccinea, mediante a abundância dela no local. Estas informações irão beneficiar diretamente a conservação e restauração de assembleias biológicas com características semelhantes a este local.

Palavras-chave: Cattleya coccinea, Complexo Serra da Farofa, Drimys angustifolia, Floresta Ombrófila Mista Alto-Montana, padrão espacial agregado.

Abstract

The objective of this study was to learn about the richness and structure of epiphytic orchids and their phorophytes, to evaluate the interactions between these assemblages, and to sample host and non-host species of orchids in a remnant of Upper-Montane Araucaria Forest (FOMA). Sampling was carried out in the Serra da Farofa Complex, on a plot of 5,000 $\mathrm{m}^{2}$. Three species of orchids were sampled, colonizing 15 species of forophytes. The spatial distribution of phorophytes and orchids was aggregated. Forophytes colonized by orchids had significantly higher height and diameter at breast height (DBH) than other forophytes. Roughtype rhytidome was the most common in orchid-colonized phorophytes. Drimys angustifolia (Winteraceae) and Myrceugenia euosma (Myrtaceae) were the main forophytic species, crucial for the maintenance of orchid assemblage at this site. The information in this manuscript highlights the in-situ protection of Cattleya coccinea by its abundance at the site. This information will directly benefit the conservation and restoration of biological assemblies with characteristics like this site.
\end{abstract}

Key words: Cattleya coccinea, Serra da Farofa Complex, Drimys angustifolia, Upper-Montane Mixed Ombrophilous Forest, aggregate spatial pattern.

\footnotetext{
' Universidade do Estado de Santa Catarina, Centro de Ciências Agroveterinárias, Depto. Engenharia Florestal, Lages, SC, Brasil.

${ }^{2}$ ORCID: <https://orcid.org/0000-0003-4422-5116>.

${ }^{3}$ ORCID: $<$ https://orcid.org/0000-0002-3539-9862>.

${ }^{4}$ ORCID: $<$ https://orcid.org/0000-0003-2952-2171>.

${ }^{5}$ ORCID: $<$ https://orcid.org/0000-0002-7445-7244>.

${ }^{6}$ Autor para correspondência: karynaflorestal@yahoo.com.br
} 


\section{Introdução}

As epífitas vasculares possuem expressiva importância na biodiversidade mundial, são altamente diversificadas, compondo aproximadamente 9\% das Embriófitas atuais, compreendendo mais de 27.000 espécies, distribuídas em 73 famílias (Zotz et al. 2013). Notoriamente, abrangem, em alguns ecossistemas montanhosos, até $52 \%$ da riqueza das Traqueófitas (Kelly et al. 2004). São plantas sensíveis à umidade, dependem de uma árvore suporte para se fixarem e adquirirem nutrientes, principalmente, de fontes atmosféricas como a precipitação e as partículas que se depositam no dossel (Nadkarni \& Solano 2002; Prescott 2002; Perez et al. 2005).

Estas plantas fornecem importantes recursos e habitats para uma grande variedade de animais e microrganismos (Céréghino et al. 2019). Além disso, são fundamentais em processos ecossistêmicos nas florestas tropicais e temperadas, particularmente na ciclagem de nutrientes, capturando e retendo minerais de fontes atmosféricas e transferindo-os para outros componentes do ecossistema (Tejo et al. 2015). Estas condições fazem das epífitas excelentes bioindicadoras ecológicas de diferentes estágios sucessionais em ecossistemas florestais (TrianaMoreno et al. 2003; Zotz \& Bader 2009). Ocupam nichos especializados, sendo plantas muito sensíveis a perturbações antrópicas (Wolf 2005; Werner \& Gradstein 2009; Bianchi \& Kersten 2014) e às mudanças climáticas globais (Zotz \& Bader 2009).

As orquídeas consistem na família mais representativa da sinúsia epifítica vascular, contribuindo com $68 \%$ de todas as espécies conhecidas (Zotz et al. 2013). As Orchidaceae, assim como outras epífitas, podem demonstrar preferência por algum forófito. Esta relação pode decorrer devido a propriedades ou características do forófito, como o tipo da casca, características arquiteturais (Wagner et al. 2015) e do tamanho da árvore (Hernández-Pérez et al. 2018).

A relação entre as orquídeas epífitas e seus forófitos requerem mais estudos, especialmente, sobre os mecanismos e propriedades que controlam a distribuição de orquídeas em diferentes forófitos (Rasmussen \& Rasmussen 2018). Segundo estes autores, esta relação recebe pouca atenção na atualidade, apesar da sua relevância para a conservação de espécies raras e ameaçadas de Orchidaceae.

Na Floresta Atlântica, considerada o quarto maior hotspot mundial em número de espécies vegetais (Brooks et al. 2002) e centro neotropical de diversidade e endemismo (Ribeiro et al. 2009), as Orchidaceae representam $46,5 \%$ das epífitas vasculares (Freitas et al. 2016). Nas áreas sob influência nebular, onde a umidade do ar é elevada na maior parte do dia, as epífitas costumam ter elevada abundância (Webster 1995). Estes ecossistemas são caracterizados pelo predomínio de fatores como raridade geográfica, endemismo, distribuição reduzida de populações, além de reduzido número de polinizadores e dispersores (Lewis 1971; Stadmüller 1987; Falkenberg 2003). As florestas nebulares que ocorrem em Santa Catarina, possuem altitudes superiores a $1.000 \mathrm{~m}$, estando contidas, especialmente em remanescentes de Floresta Ombrófila Mista Alto-Montana (FOMA) (IBGE 2012).

Com base no exposto, objetivou-se conhecer a riqueza e estrutura de orquídeas epífitas (vertical e horizontal) e seus forófitos (horizontal), as interações entre estas duas assembleias, bem como, características de hospedeiros e não hospedeiros em um remanescente de Floresta Ombrófila Mista sob influência nebular no Planalto Serrano Catarinense.

Como hipótese acredita-se que árvores com características que facilitem o estabelecimento e crescimento de epífitas, como maior DAP e casca rugosa, terão maior abundância de orquídeas epífitas.

\section{Material e Métodos}

\section{Área de estudo}

$O$ estudo foi conduzido em um remanescente de Floresta Ombrófila Mista Alto-Montana (IBGE 2012), sob influência nebular, no município de Urupema, Santa Catarina, Brasil. Trata-se de uma Reserva Particular do Patrimônio Natural Estadual (RPPNE) pertencente à empresa Klabin S.A., a qual denomina-se Complexo Serra da Farofa, composta por seis blocos distintos. A RPPNE possui 4.965,86 ha que compõem parte da bacia hidrográfica do Rio Canoas. A área de estudo localiza-se no Bloco I (Fig. 1), com 1.367,43 ha, designada de fazenda das

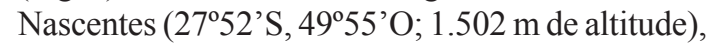
que compreende quatro das cinco nascentes que formam o Rio Caveiras, que abastece a cidade de Lages, principal munícipio do Planalto Serrano Catarinense.

O clima da região é do tipo " $\mathrm{Cfb}$ ", classificado como temperado úmido (Köppen 1948), com chuvas distribuídas durante todo o ano e precipitação média anual de $1.634 \mathrm{~mm}$. A temperatura média anual é de $14,1^{\circ} \mathrm{C}$ (Climate-data 2018), sendo comum a ocorrência de geadas e neve nos meses mais frios. 


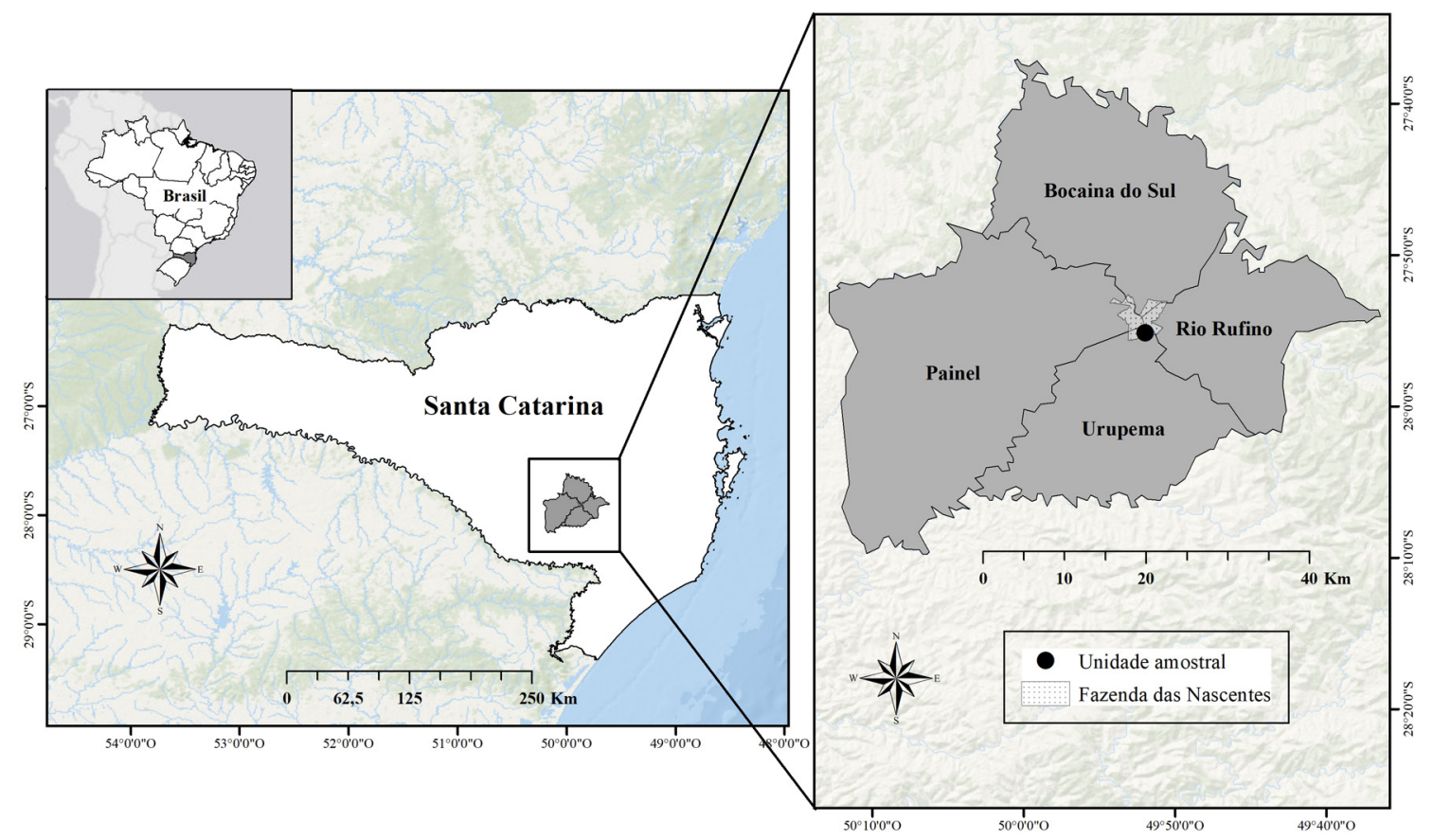

Figura 1 - Localização da área de estudo, Fazenda das Nascentes, Planalto Serrano Catarinense, Brasil. Figure 1 - Location of the study area, Fazenda das Nascentes, Southern Plateau of Santa Catarina, Brazil.

\section{Amostragem}

Os dados foram amostrados no período de janeiro de 2015 a janeiro de 2016, em uma parcela permanente de $100 \times 50 \mathrm{~m}\left(5.000 \mathrm{~m}^{2}\right)$, dividida em subparcelas de $10 \times 10 \mathrm{~m}\left(100 \mathrm{~m}^{2}\right)$. Foi identificada toda a assembleia arbórea, além de, mensuradas as alturas e Circunferências a Altura do Peito (CAP) (indivíduos $\geq 15 \mathrm{~cm}$ de CAP), medidas que, posteriormente, foram transformadas em Diâmetro a Altura do Peito (DAP). Foram então selecionados, exclusivamente, os forófitos de Orchidaceae, dividindo-os em seis estratos verticais (I a VI). Os cinco primeiros estratos $(\mathrm{I}-\mathrm{V})$ corresponderam a intervalos verticais de um metro cada (1 a $5 \mathrm{~m}$ ), já o último (VI) foi equivalente a parte mais externa da copa de cada forófito. A altura da primeira bifurcação, na maior parte dos forófitos, correspondeu aos estratos II e III. Verificou-se a riqueza e a abundância das orquídeas em cada extrato vertical (I-VI) em suas árvores suporte. A avaliação das orquídeas foi realizada por meio de binóculos e visualização a olho nu. A quantificação da abundância das espécies de orquídeas foi pela ocorrência de agrupamentos formados (touceiras) (Fontoura et al. 2009). Também foi fotografado e realizado a classificação do tipo de ritidoma de cada espécie forofítica, de acordo com Ribeiro et al. (1999).
As espécies de Orchidaceae e forófitos foram identificadas com auxílio de literatura especializada. A grafia dos nomes científicos foi conferida segundo Brummitt \& Powell (1992). Os materiais férteis foram incorporados ao Herbário LUSC (Herbário Lages da Universidade do Estado de Santa Catarina), com sede no Centro de Ciências Agroveterinárias, em Lages-SC.

\section{Análise de dados}

Para análise da estrutura populacional das espécies de Orchidaceae foi calculada a Densidade Absoluta (DA). Verificou-se, pelo teste de proporção $(p \leq 0,05)$, se haviam diferenças significativas entre a abundância de cada espécie epífita ao longo dos diferentes estratos verticais nos forófitos.

A estrutura horizontal da comunidade forofítica foi determinada pelos descritores fitossociológicos: Densidade Absoluta (DA), Dominância Absoluta (DoA) e Frequência Absoluta (FA). Para as duas espécies de hospedeiros mais representativos foi determinada a distribuição espacial pelo Índice de Morisita Padronizado (IM) (Ludwig \& Reynolds 1988). Também foi determinado o padrão espacial para as espécies de orquídeas, de acordo com os estratos de ocorrência. Os estratos, para cada espécie, que compreenderam menos que cinco indivíduos, 
não foram submetidas a análise de distribuição espacial, por não atenderem as premissas da análise. Os padrões de classificação considerados foram: aleatório $(-0,5$ a 0,5$)$, regular $(<-0,5)$ e agregado $(>0,5)$ (Hay et al. 2000).

Foram testadas se haviam diferenças significativas entre as alturas e diâmetros das espécies de forófitos e não forofíticas. Por meio do teste de Shapiro-Wilk foi testada a normalidade destes dados, sendo que os mesmos foram nãoparamétricos. Portanto, foi realizado o teste de $U$ de Mann-Whitney.

Todas as análises foram realizadas no programa estatístico RStudio Team (2015), com auxílio da biblioteca Vegan (Oksanen et al. 2018).

\section{Resultados}

Riqueza e estrutura de forófitos e orquídeas

Foram amostrados 899 indivíduos arbóreos (DA 1.798 ind.ha $^{-1}$ ), pertencentes a 24 espécies. Destes, 237 indivíduos (DA 474 ind.ha $^{-1}$ ) consistiram em forófitos de Orchidaceae, os quais, compreenderam 15 espécies, distribuídas em 15 gêneros e 12 famílias botânicas (Tab. 1). Lauraceae foi a família forofítica mais representativa com três espécies, seguida por Myrtaceae com duas espécies, as demais famílias foram representadas por uma espécie cada. Os forófitos mais abundantes foram Drimys angustifolia (Winteraceae) com 117 indivíduos (DA 234 ind.ha $^{-1}$ ), Myrceugenia euosma (Myrtaceae) com 57 indivíduos (DA 114 ind.ha ${ }^{-1}$ ) e Weinmannia humilis (Cunoniaceae) 17 indivíduos (DA 34 ind.ha $^{-1}$ ). Myrceugenia euosma foi a espécie com maior área basal $\left(43,06 \mathrm{~m}^{2} . \mathrm{ha}^{-1}\right)$ e a mais bem distribuída na área de amostragem.

Em relação as orquídeas epífitas, foram registrados 848 indivíduos (DA de 1.696 ind.ha $^{-1}$ ), pertencentes a três espécies: Cattleya coccinea Lindl. com 752 indivíduos (DA de 1.504 ind.ha $^{-1}$ ); Gomesa gomezoides (Barb.Rodr.) Pabst com 45 indivíduos (DA de 90 ind.ha ${ }^{-1}$ ) e; Phymatidium microphyllum var. herteri (Schltr.) Toscano com 51 indivíduos (DA de 102 ind.ha ${ }^{-1}$ ) (Tab. 1).

Tabela 1 - Forófitos de Orchidaceae em remanescente de Floresta Ombrófila Mista sob influência nebular, Planalto Serrano Catarinense e respectivos valores de Densidade absoluta (DA), Dominância absoluta (DoA), Frequência absoluta (FA) e tipo de ritidoma.

Table 1 - Forophytes of Orchidaceae in Upper-Montane Araucaria Forest with nebular influence, Southern Plateau, Santa Catarina and their respective Absolute Density (DA), Absolute dominance (DoA), Absolute frequency (FA) and type of rhytidoma.

\begin{tabular}{|c|c|c|c|c|c|}
\hline Família & Espécie & DA* & DoA* & FA* & $\begin{array}{c}\text { Tipo de } \\
\text { Ritidoma }\end{array}$ \\
\hline Winteraceae & Drimys angustifolia Miers & 234 & 40,37 & 46 & rugoso \\
\hline Myrtaceae & Myrceugenia euosma (O.Berg) D.Legrand & 114 & 43,06 & 50 & escamoso \\
\hline Cunoniaceae & Weinmannia humilis Engl. & 34 & 32,44 & 24 & fissurado \\
\hline Lauraceae & Cinnamomum amoenum (Nees \& Mart.) Kosterm. & 14 & 17,30 & 12 & escamoso \\
\hline Aquifoliaceae & Ilex paraguariensis A.St.-Hil. & 22 & 5,39 & 18 & rugoso \\
\hline Myrtaceae & Siphoneugena reitzii D.Legrand & 18 & 2,43 & 16 & laminado \\
\hline Araucariaceae & Araucaria angustifolia (Bertol.) Kuntze & 8 & 6,61 & 8 & fissurado \\
\hline Dicksoniaceae & Dicksonia sellowiana Hook. & 8 & 2,53 & 6 & fibroso \\
\hline Rosaceae & Prunus myrtifolia (L.) Urb. & 4 & 1,74 & 4 & rugoso \\
\hline Lauraceae & Ocotea pulchella (Nees \& Mart.) Mez & 4 & 1,02 & 4 & lenticelado \\
\hline Lauraceae & Persea willdenovii Kosterm. & 4 & 2,18 & 2 & escamoso \\
\hline Symplocaceae & Symplocos tetrandra Mart. & 4 & 0,41 & 4 & rugoso \\
\hline Proteaceae & Roupala montana var. brasiliensis (Klotzsch) K.S.Edwards & 2 & 0,46 & 2 & rugoso \\
\hline Fabaceae & Mimosa scabrella Benth. & 2 & 0,15 & 2 & reticulado \\
\hline Asteraceae & Vernonanthura discolor (Spreng.) H.Rob. & 2 & 0,10 & 2 & lenticelado \\
\hline
\end{tabular}

* DA (ind.ha $\left.{ }^{-1}\right) ; \operatorname{DoA}\left(\mathrm{m}^{2} \cdot \mathrm{ha}^{-1}\right) ; \mathrm{FA}(\%)$. 


\section{Distribuição espacial de forófitos e orquídeas}

O padrão de distribuição espacial das espécies forofíticas mais abundantes e orquídeas por estrato foi agregado. Drimys angustifolia apresentou um IM de 0,53 e M. euosma IM de 0,50. Já para C. coccinea foram verificados os padrões de distribuição em todos os estratos I (IM 0,51), II (IM 0,52), III (IM 0,54), IV (IM 0,52), V (IM 0,51) e VI (IM 0,53). Para G. gomezoides foi possível conhecer IM dos estratos I (IM 0,54), II (IM 0,55), III (IM 0,53) e IV (IM 0,55) e em $P$. microphyllum var. herteri foram obtidos, apenas, valores dos estratos II (IM 0,52) e III (IM 0,54).

\section{Características de forófitos e não forófitos}

A altura média total da comunidade arbórea do remanescente foi de 7,03 m ( $\pm 2,66 \mathrm{~m})$ e o Diâmetro a Altura do Peito (DAP) médio de 14,49 cm $( \pm 9,20$ $\mathrm{cm})$. Já os indivíduos arbóreos que foram forófitos de orquídeas apresentaram altura média de 8,24 m $( \pm 2,65 \mathrm{~m})$ e DAP médio de $17,36 \mathrm{~cm}( \pm 10,99 \mathrm{~cm})$ e os indivíduos arbóreos que não foram colonizados por orquídeas, tiveram uma altura média de $6,59 \mathrm{~m}$ $( \pm 2,52 \mathrm{~m})$ e DAP médio de $13,46 \mathrm{~cm}( \pm 8,24 \mathrm{~cm})$. Houve diferenças significativas, entre os valores das alturas médias ( $U$ de Mann-Whitney $50867 p<$ 0,0001) e DAP médio ( $U$ de Mann-Whitney 60164 $p<0,0001)$ entre forófitos e não forófitos.

Para os forófitos, a classe diamétrica de DAP de 10 a $19 \mathrm{~cm}$ foi a mais representativa na colonização de orquídeas (Fig. 2). Já os indivíduos arbóreos que não foram colonizadas por orquídeas, ficaram comtemplados, especialmente, na classe de DAP de 0 a $9 \mathrm{~cm}$. Os indivíduos enquadrados nas maiores classes diamétricas, em geral foram colonizados por orquídeas.

\section{Relações entre orquídeas e forófitos}

As espécies $D$. angustifolia e $M$. euosma foram as mais abundantes na área de estudo, assim como, os principais forófitos de Orchidaceae. Em relação ao total de indivíduos encontrados na área de estudo, $66,1 \%$ dos indivíduos de D. angustifolia e 40,1\% dos de $M$. euosma foram forófitos de orquídeas. Estas duas espécies de forófitos (D. angustifolia $49,4 \%$ e $M$. euosma $24,1 \%$ ) representaram $73,5 \%$ do total de forófitos de orquídeas neste estudo (Tab. 1). Drimys angustifolia foi colonizada por todas as três orquídeas, já, M. euosma não foi colonizada por G. gomezoides.

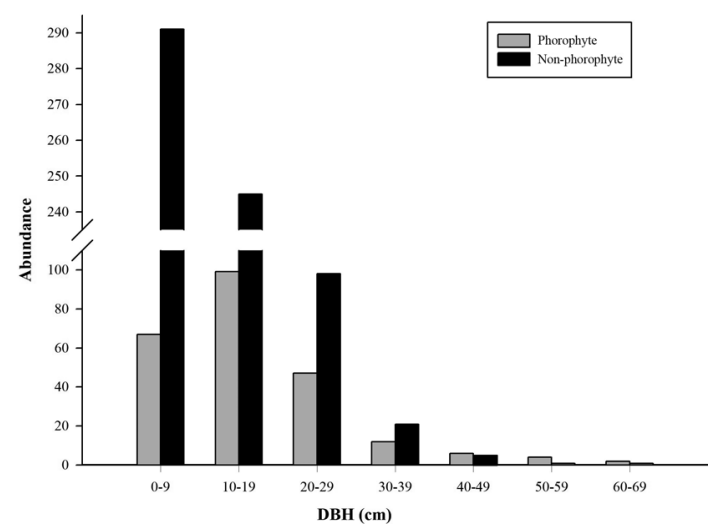

Figura 2 - Número de indivíduos arbóreos (Abundance) por classes de diâmetro $(\mathrm{DBH})$ de forófitos e não forófitos de Orchidaceae em um remanescente de Floresta Ombrófila Mista sob influência nebular, Planalto Serrano Catarinense.

Figure 2 - Number of arboreal individuals (Abundance) by diameter classes (DBH) of phorophytes and non-phorophytes of Orchidaceae in a remnant of Upper-Montane Araucaria Forest with nebular influence, Southern Plateau, Santa Catarina.

Nenhuma das orquídeas colonizou todas as espécies de forófitos. Cattleya coccinea colonizou o maior número de espécies, 14 das 15 espécies forofíticas, exceto Mimosa scabrella (Fabaceae). Gomesa gomezoides colonizou sete espécies forofíticas e P. microphyllum var. herteri foi verificada em cinco espécies de forófitos.

Em relação a distribuição vertical das espécies de orquídeas nos forófitos (Fig. 3), C. coccinea e $G$. gomezoides foram observadas em todos os estratos (I-VI), enquanto, P. microphyllum var. herteri, não foi verificada na copa superior (VI). Em relação a abundância das orquídeas nos diferentes estratos, houve diferenças significativas, entre estratos, para todas as três espécies, como evidenciado na Figura 3.

Em relação ao ritidoma dos forófitos de Orchidaceae (Tab. 1) (Fig. 4) do tipo rugoso foi o mais comum, ocorrendo em cinco espécies $(33,33 \%)$. Seguidos pelos tipos escamoso com três espécies e fissurado e lenticelado com duas espécies cada. Os tipos de ritidomas laminado, fibroso e reticulado corresponderam a uma única espécie cada.

\section{Discussão}

\section{Riqueza e estrutura de forófitos} e orquídeas

A hipótese elencada neste estudo foi aceita. Os forófitos de D. angustifolia e $M$. euosma, os mais abundantes neste estudo, colonizados pelas 


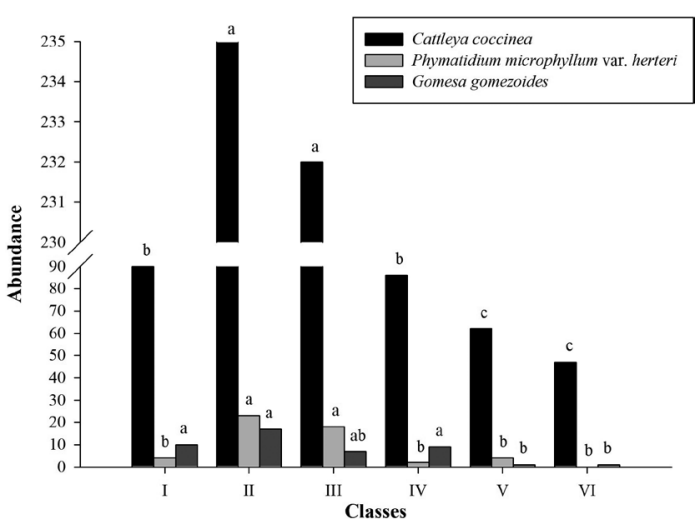

Figura 3 - Abundância de três espécies de Orchidaceae por estratos verticais em forófitos em um remanescente de Floresta Ombrófila Mista sob influência nebular, Planalto Serrano Catarinense (as letras representam as diferenças significativas pelo Teste de Proporções, a $95 \%$ de probabilidade).

Figure 3 - Abundance of three species of Orchidaceae in vertical classes in phorophyte in a remnant of Upper-Montane Araucaria Forest with nebular influence, Southern Plateau, Santa Catarina (the letters represent the significant differences by the Proportion Test at $95 \%$ probability).

três espécies de orquídeas, são comuns na região, como observado por Higuchi et al. (2013) em remanescente próximo ao local de estudo, onde, $M$. euosma caracterizou-se como a espécie com maior DA. Martins et al. (2012) também verificaram elevada abundância destas duas espécies em diferentes áreas, neste mesmo bloco da RPPNE.

A área estudada apresentou uma baixa riqueza de orquídeas em comparação a outros estudos realizados em FOM, no Brasil (Kersten \& Silva 2002; Borgo \& Silva 2003; Buzatto et al. 2008; Bonnet et al. 2009; Kersten et al. 2009; Kersten \& Kuniyoshi 2009; Furtado \& Menini-Neto 2016). Inclusive quando comparado a estudo realizado na mesma região (Planalto Serrano Catarinense), em outros remanescentes, a riqueza foi inferior (Montibeller-Silva 2018). Zhang et al. (2015) expuseram, em seu trabalho, que a riqueza de orquídeas diminui em função da elevação altitudinal, sendo que, o limite da maior expressividade da riqueza está compreendida em locais com altitude média de 1.300 metros, temperaturas médias anuais entre $13,7^{\circ} \mathrm{C}$ e 17,7 ${ }^{\circ} \mathrm{C}$ e precipitação média anual de 1.237 a 1.414 $\mathrm{mm}$. Além destes fatores, locais com elevada riqueza de epífitas, em geral, estão livres de perturbações antrópicas, encontrando-se em estágios sucessionais avançados (Triana-Moreno et al. 2003; Zotz \& Bader 2009). A referida área de estudo, localiza-se em uma RPPNE, onde as condições ambientais (altitude, temperatura média e precipitação anual) são similares às elencadas por Zhang et al. (2015), como condições ideais para uma elevada riqueza. Além do que, desde 2007 a área encontra-se completamente protegida sem interferências antrópicas, correspondendo a uma floresta em estágio sucessional de médio para avançado, entretanto, estas condições não foram suficientes para haver um maior número de espécies de orquídeas.

Neste caso, sugerimos que algum filtro ambiental possa estar limitando o incremento de espécies de orquídeas. Em geral, os filtros ambientais são os principais fatores limitantes à ocorrência da flora local, mostrando efeitos substanciais, tanto, sobre a diversidade alfa, quanto, a beta (Santos et al. 2015). Neste ecossistema, a baixa riqueza pode ter sido influenciada por um conjunto de fatores ambientais intrínsecos, como a face de exposição solar (noroeste), além da direção e velocidade dos ventos. Além disso, a matriz de paisagem (fator geográfico) pode ter restrição no fluxo de propágulos, pois, apesar do local de estudo estar inserido em uma RPPNE, que compõe uma área expressiva de floresta e campos nativos (seis blocos), estas não estão completamente conectadas. Fatores como a aleatoriedade no fornecimento de propágulos, também podem ter influenciado a baixa composição florística (Wolf 1994).

Já a C. coccinea, pode estar sendo favorecida por algum filtro ambiental, em vista a expressiva abundância e dominância ecológica que a espécie demonstrou. Cattleya coccinea é comum em FOM, em altitudes superiores a $800 \mathrm{~m}$, onde há elevada umidade pela exposição à nevoeiros (Buzatto et al. 2010). Fato que corrobora com a área estudada, que demonstra condições ideais para o seu desenvolvimento.

\section{Distribuição espacial de forófitos e orquídeas}

O padrão de distribuição de uma espécie pode variar, de acordo com a escala espacial e estágio ontogenético, o qual, encontra-se, dependendo diretamente da dinâmica da comunidade. O padrão agregado, verificado para os dois forófitos mais abundantes, provavelmente, deve-se à dispersão restrita das orquídeas, onde as sementes são depositadas próximas a planta mãe, estabelecendose, prioritariamente, nas suas proximidades (Trapnell et al. 2004; Fajardo et al. 2015). 

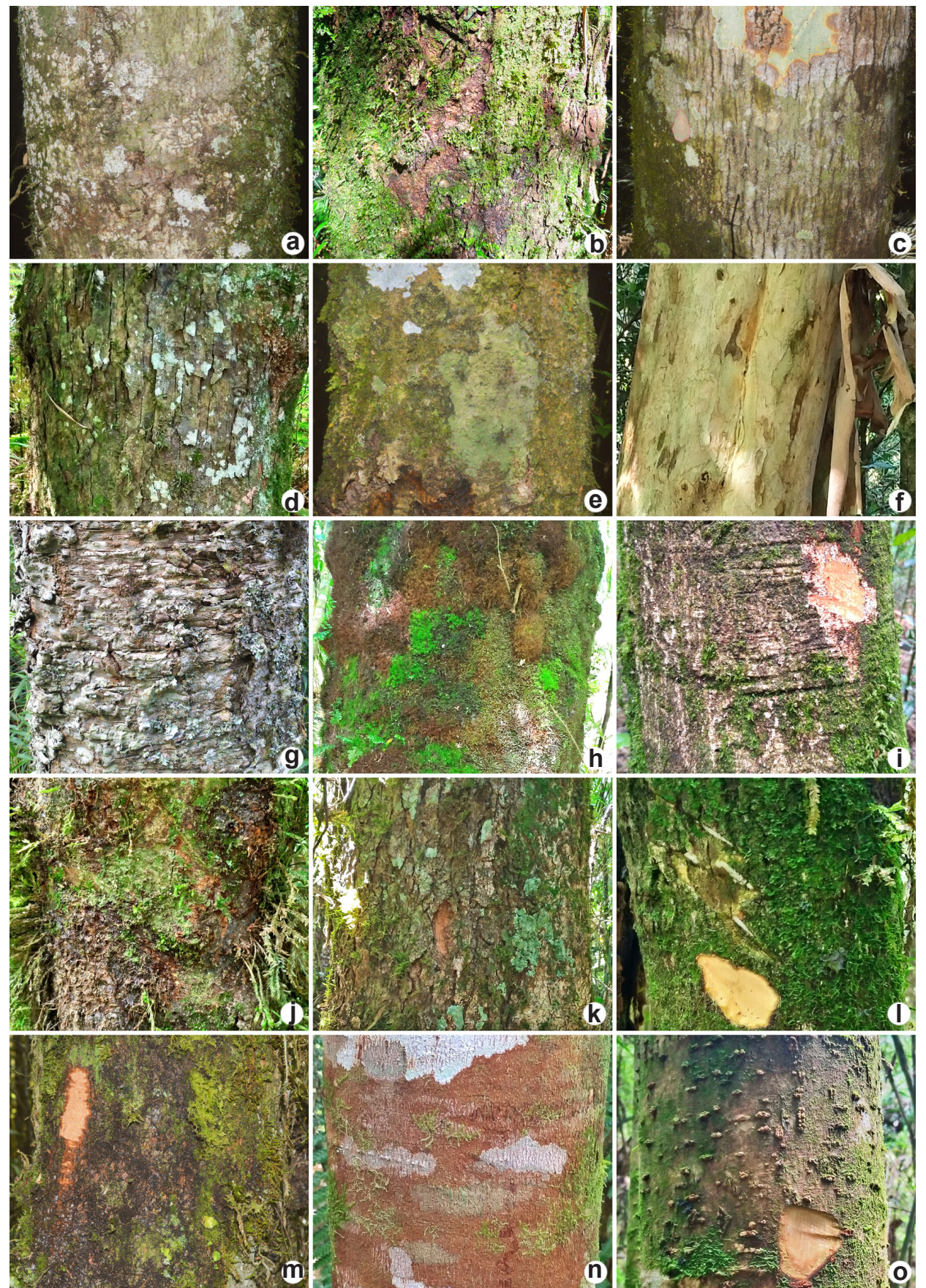

Figura 4 - a-o. Casca externa dos forófitos - a. Drimys angustifolia (Winteraceae); b. Myrcengenia euosma (Myrtaceae); c. Weinmannia humilis (Cunoniaceae); d. Cinnamomum amoenum (Lauraceae); e. Ilex paraguariensis (Aquifoliaceae); f. Siphoneugena reitzii (Myrtaceae); g. Araucaria angustifolia (Araucariaceae); h. Dicksonia sellowiana (Dicksoniaceae); i. Prunus myrtifolia (Rosaceae); j. Ocotea pulchella (Lauraceae); k. Persea willdenovii (Lauraceae); 1. Symplocos tetrandra (Symplocaceae); m. Roupala montana var. brasiliensis (Proteaceae); n. Mimosa scabrella (Fabaceae); o. Vernonanthura discolor (Asteraceae).

Figure 4 - a-o. Rhytidoma of phorophytes - a. Drimys angustifolia (Winteraceae); b. Myrcengenia euosma (Myrtaceae); c. Weinmannia humilis (Cunoniaceae); d. Cinnamomum атоеnum (Lauraceae); e. Ilex paraguariensis (Aquifoliaceae); f. Siphonengena reitzii (Myrtaceae); g. Araucaria angustifolia (Araucariaceae); h. Dicksonia sellowiana (Dicksoniaceae); i. Prunus myrtifolia (Rosaceae); j. Ocotea pulchella (Lauraceae); k. Persea willdenovii (Lauraceae); 1. Symplocos tetrandra (Symplocaceae); m. Roupala montana var. brasiliensis (Proteaceae); n. Mimosa scabrella (Fabaceae); o. Vernonanthura discolor (Asteraceae). 
Em relação ao padrão de distribuição agregado das três orquídeas, em todos os estratos verticais avaliados, é comum verificar este padrão para espécies epífitas (Nieder et al. 2000; Burns \& Zotz 2010). O micro-habitat favorável (umidade e luminosidade) (Woods et al. 2015; Padilha et al. 2017), assim como, a necessidade de interações com fungos específicos (Otero et al. 2007) essenciais para a germinação das sementes das orquídeas e a dispersão limitada são fatores determinantes no sucesso reprodutivo ser maior próximo a planta mãe. Infelizmente, o padrão agregado pode ser categorizado como crítico, visto que, as espécies tornam-se mais vulneráveis a ações predatórias (Fajardo et al. 2015), por outro lado, o uso sustentável é facilitado.

\section{Características de forófitos e não forófitos}

Neste estudo, os forófitos de orquídeas caracterizaram-se por apresentar médias de altura e DAP, significativamente maiores em comparação aos não forófitos. Zotz \& Vollrath (2003) afirmam que a ocupação epifítica, assim como, a abundância por forófito, correlacionam-se axiomaticamente, com a idade da árvore e área do substrato. Existindo assim, uma especificidade entre orquídeas e altura e diâmetro do forófito (Hernández-Pérez et al. 2018). À medida que as árvores crescem, tanto em diâmetro, quanto em altura, contribuem para mudanças na estrutura da sinúsia epifítica, aumentando a heterogeneidade de micro-hábitats, especialmente, dentro das copas (Woods et al. 2015). Wang et al. (2016) observaram que a riqueza de epífitas aumenta em relação ao ganho de diâmetro do hospedeiro, em florestas tropicais nebulares. Assim como, neste estudo, árvores com DAP superiores foram, em sua maioria, colonizadas por orquídeas epífitas.

\section{Relações entre orquídeas e forófitos}

As espécies arbóreas (D. angustifolia e $M$. euosma) mais colonizadas pelas orquídeas, são evidenciadas como comuns na região, típicas de formações de FOMA. Em levantamento realizado próximo ao local de estudo, M. euosma foi a espécie com maior DA (DA 326 ind.ha-1), já $D$. angustifolia (DA 46 ind.ha $^{-1}$ ) apresentou menor representatividade (Higuchi et al. 2013). Martins et al. (2012) também verificaram elevada DA de D. angustifolia (DA 235 ind.ha $^{-1}$ ) e M. euosma (DA 315 ind.ha $^{-1}$ ) em diferentes áreas desta mesma RPPNE.
Em estudo sobre relações entre orquídeas e forófitos, Migenis \& Ackerman (1993), também elencaram duas espécies arbóreas forofíticas, como muito representativas, estas duas, conjuntamente, representaram $62,9 \%$ das árvores suporte de orquídeas em uma área de Porto Rico. Semelhantes aos resultados verificados neste trabalho. Cabe ressaltar que os autores, citados anteriormente, também verificaram uma baixa riqueza de orquídeas em seu levantamento, mas neste caso, associaram, principalmente, a degradações antrópicas.

Em relação a distribuição vertical das três Orchidaceae nos forófitos, G. gomezoides caracterizou-se por ser uma espécie mais tolerante às variações de umidade e luminosidade, encontrando-se distribuída mais uniformemente nos quatro primeiros estratos. Enquanto $C$. coccinea apresentou três níveis de ocorrência, relacionadas ao número de indivíduos, sendo significativamente mais abundante nos estratos II e III. P. microphyllum var. herteri seguiu o mesmo padrão da anterior, onde os estratos II e III apresentaram diferenças significativa em número de indivíduos, sendo as duas principais zonas de colonização.

Os estratos II e III, que corresponderam, na maioria dos forófitos, ao final do tronco e início dos ramos, apresentaram a maior abundância, especialmente, de $C$. coccinea e $P$. microphyllum var. herteri. Esta abundância pode estar correlacionada, principalmente, a menor incidência luminosa, além da proteção contra geadas e, até mesmo, de possíveis ocorrências de neve e chuva congelada, fenômenos comuns no local. Já a micro-orquídea P. microphyllum var. herteri, que não foi verificada no último extrato (copa superior) dos forófitos e também, raramente foi observada em áreas de borda do remanescente, fator condicionado, provavelmente, a mesma não possuir órgãos de reserva, estando mais susceptível em áreas ensolaradas e com menor unidade relativa do ar. Esta espécie, por muitas vezes, ocorreu em árvores jovens, e de DAP reduzido, assim como observado por Royer et al. (2014). Esta espécie também foi verificada colonizando caule (colmo) de taquaras, em locais adjacentes a área de estudo. Estas variações de abundância, na estratificação vertical, ou preferências por zonas do forófito são, comumente, evidenciadas em outros estudos sobre epífitos (Zimmerman \& Olmestd 1992; Rogalski \& Zanin 2003; Kersten \& Silva 2001; HernándezPérez et al. 2018). 
Estudos em assembleias de epífitas descrevem que troncos rugosos têm maiores probabilidades de ocupação (Callaway et al. 2002; Medeiros et al. 2014). Entretanto, outros autores elencaram ritidomas fissurados como as mais importantes na colonização de orquídeas, enfatizando que a espessura é fundamental (quanto mais espessa, maior a colonização) (Hernández-Pérez et al. 2018). Características presentes no ritidoma rugoso facilitam o acúmulo de material orgânico e água, recursos fundamentais para ampliar o sucesso de colonização e o estabelecimento das epífitas (Callaway et al. 2002; Medeiros et al. 2014; Wagner et al. 2015). Já ritidomas mais grossos, oferecem uma melhor base de apoio, dificultando o desprendimento de sementes e plantas (Wagner et al. 2015).

Apesar do exposto, neste estudo, observou-se forófitos com ritidoma rugoso/fissurado que não foram colonizadas por orquídeas, como Piptocarpha angustifolia Dusén ex Malme (Asteraceae). Contudo, trata-se de uma espécie pioneira de ciclo de vida curto, que coloniza as bordas de remanescentes. $\mathrm{Na}$ região raramente observou-se a colonização de orquídeas em forófitos desta classificação ecológica (observação pessoal). Há outros casos em que, devido a química da casca, sobretudo pela presença de taninos (Callaway et al. 2002), espécies arbóreas de estágios sucessionais mais avançados que possuem ritidoma rugoso e suas variações, configuram habitat desfavorável às Orchidaceae.

Como considerações, recomendamos a realização de mais estudos sobre riqueza e estrutura de assembleias epifíticas, bem como, sobre as relações existentes entre estas e seus forófitos nas diferentes fisionomias da FOM. Na área em questão, salientamos que estudos futuros poderão elucidar quais filtros ambientais limitam a riqueza de orquídeas e facilitam a abundância de $C$. coccinea. Novos trabalhos serão fundamentais para conservação in situ, assim como para a criação de estratégias e ações prioritárias para a conservação. As espécies forofíticas mais abundantes $(D$. angustifolia e $M$. euosma), são cruciais para a manutenção da assembleia de orquídeas do Bloco I da RPPNE Complexo Serra da Farofa e regiões adjacentes. As informações contidas neste estudo elucidam caraterísticas e espécies de forófitos para restauração ecológica, utilizando as orquídeas levantadas, ou até mesmo criação de habitats melhorados por estas espécies. As informações deste manuscrito destacam a proteção in situ de $C$. coccinea, visto a abundância dela no local, a qual não é verificada para esta espécie em outros locais semelhantes na mesma região (observação pessoal). Estas informações irão beneficiar diretamente a conservação e restauração de assembleias biológicas com características semelhantes.

\section{Agradecimentos}

Agradecemos à Universidade do Estado de Santa Catarina (UDESC), o ensino de qualidade; à Fundação de Apoio à Manutenção e ao Desenvolvimento da Educação Superior (FUMDES), a concessão da bolsa de Doutorado à primeira autora; à empresa Klabin $\mathrm{S} / \mathrm{A}$, o apoio financeiro e a disponibilização da área para realização do estudo; aos pesquisadores Lilian Iara Bet Stedille e Newton Clóvis Freitas Costa, as contribuições na redação do manuscrito, bem como aos revisores anônimos, as sugestões.

\section{Referências}

Bianchi JS \& Kersten RA (2014) Edge effect on vascular epiphytes in a subtropical Atlantic Forest. Acta Botanica Brasilica 28: 120-126.

Bonnet A, Lavoranti OJ \& Curcio GR (2009) Epífitos vasculares no Corredor de Biodiversidade Araucária, bacia do Rio Iguaçú, Paraná, Brasil. Cadernos da Biodiversidade 6: 49-70.

Borgo M \& Silva SM (2003) Epífitos vasculares em fragmentos de Floresta Ombrófila Mista, Curitiba, Paraná, Brasil. Revista Brasileira de Botânica 26: 391-401.

Brooks TM, Mittermeier RA, Mittermeier CG, Fonseca GA, Rylands AB, Konstant WR, Flick P, Pilgrim J, Oldfield S, Magin G \& Hilton-Taylor C (2002) Habitat loss and extinction in the hotspots of biodiversity. Conservation Biology 16: 909-923.

Brummitt RK \& Powell CE (1992) Authors of plant names: a list of authors of scientific names of plants, with recommended standard form of their names including abbreviations. Royal Botanic Gardens, Kew. 732p.

Burns KC \& Zotz G (2010) A hierarchical framework for investigating epiphyte assemblages: networks, metacommunities and scale. Ecology 91: 377-385.

Buzatto CR, Severo BMA \& Waechter JL (2008) Composição florística e distribuição ecológica de epífitos vasculares na Floresta Nacional de Passo Fundo, Rio Grande do Sul. Iheringia, série Botânica 63: 231-239.

Buzatto CR, Ferreira PPA, Welker CAD, Seger GDS, Hertzog A \& Singer RB (2010) O gênero Cattleya Lindl. (Orchidaceae: Laeliinae) no Rio Grande do Sul, Brasil. Revista Brasileira de Biociências 8: 388-398.

Callaway RM, Reinhart KO, Moore GW, Moore DJ \& Pennings SC (2002) Epiphyte host preferences and host traits: mechanisms for species-specific interactions. Oecologia 132: 221-230. 
Céréghino R, Corbara B, Hénaut Y, Bonhomme C, Compin A \& Dejean A (2019) Ant and spider species as surrogates for functional community composition of epiphyte-associated invertebrates in a tropical moist forest. Ecological Indicators 96: 694-700.

Climate-data (2018) Dados climáticos para cidades mundiais. Disponível em $<$ http://pt.climate-data. org > . Acesso em 13 março 2018.

Fajardo CG, Costa RA, Vieira FA \& Molina WF (2015) Distribuição espacial de Cattleya granulosa Lindl.: uma orquídea ameaçada de extinção. Floresta Ambiente 22: 164-170.

Falkenberg DB (2003) Matinhas nebulares e vegetação rupícola dos Aparatos da Serra Geral (SC/RS), sul do Brasil. Tese de Doutorado. Universidade Estadual de Campinas, Campinas. 594p.

Fontoura T, Rocca MA, Schilling AC \& Reinert F (2009) Epífitas da floresta seca da Reserva Ecológica Estadual de Jacarepiá, sudeste do Brasil: relações com a comunidade arbórea. Rodriguésia 60: 171-185.

Freitas L, Salino A, Menini Neto L, Almeida TE, Mortara SR, Stehmann JR, Amorim AM, Guimarães EF, Coelho MN, Zanin A \& Forzza RC (2016) A comprehensive checklist of vascular epiphytes of the Atlantic Forest reveals outstanding endemic rates. PhytoKeys 58: 65-79.

Furtado SG \& Menini-Neto L (2016) Vascular epiphytic flora of a high montane environment of Brazilian Atlantic Forest: composition and floristic relationships with other ombrophilous forests. Acta Botanica Brasilica 30: 422-436.

Hay JD, Bezerril MX, Calouro AM, Costa EMN, Ferreira AA, Gastal MLA, Goes Junior CD, Manzan DJ, Martins CR, Monteiro JMG, Oliveira SA, Rodrigues MCM, Seyffarth JAS \& Walter BMT (2000) Comparação do padrão da distribuição espacial em escalas diferentes de espécies nativas do cerrado, em Brasília, DF. Revista Brasileira de Botânica 23: 341-347.

Hernández-Pérez E, Solano E \& Ríos-Gómez R (2018) Host affinity and vertical distribution of epiphytic orchids in a montane cloud forest in southern Mexico. Botanical Sciences 96: 200-217.

Higuchi P, Silva AC, Almeida JA, Bortoluzzi RLC, Mantovani A, Ferreira TS, Souza ST, Gomes J P \& Silva KM (2013) Florística e estrutura do componente arbóreo e análise ambiental de um fragmento de Floresta Ombrófila Mista AltoMontana no município de Painel, SC. Ciência Florestal 23: 153-164.

IBGE - Instituto Brasileiro de Geografia e Estatística (2012) Manual técnico da vegetação brasileira. IBGE, Rio de Janeiro. 270p.

Kelly DL, O’Donovan G, Feehan J, Murphy S, Drangeid SO \& Marcano-Berti L (2004) The epiphyte communities of a montane rain forest in the Andes of Venezuela: patterns in the distribution of the flora. Journal of Tropical Ecology 20: 643-666.
Kersten RA \& Kuniyoshi YS (2009) Conservação das florestas na bacia do alto Iguaçu, Paraná - avaliação da comunidade de epífitas vasculares em diferentes estágios serais. Floresta 39: 51-66.

Kersten RA \& Silva SM (2001) Composição florística e distribuição espacial de epífitas vasculares em floresta da planície litorânea da Ilha do Mel, Paraná, Brasil. Revista Brasileira de Botânica 24: 213-226.

Kersten RA \& Silva SM (2002) Florística e estrutura do componente epifítico vascular em floresta ombrófila mista aluvial do rio Barigüi, Paraná, Brasil. Revista Brasileira de Botânica 25: 59-267.

Kersten RA, Kuniyoshi YS \& Roderjan CV (2009) Comunidade epífita em duas formações florestais do Rio São Jerônimo, Bacia do Rio Iguaçú, municípios de Guarapuava e Pinhão, Paraná. Iheringia, série Botânica 64: 33-43.

Köppen W (1948) Climatologia: con un estudio de los climas de la tierra. Fondo de Cultura Econômica, México D.F. 479p.

Lewis WH (1971) High floristic endemism in Low Cloud Forests of Panamá. Biotropica 3: 78-80.

Ludwig JA \& Reynolds JF (1988) Statistical Ecology: a primer on methods and computing. John Wiley \& Sons, New York. 337p.

Martins D, Rodrigues AL, Chaves CL, Mantovani A \& Bortoluzzi RLC (2012) Estrutura de um remanescente de Floresta Ombrófila Mista em Urupema, Santa Catarina, Brasil. Revista de Ciências Agroveterinárias 11: 126-137.

Medeiros TDS, Jardim MAG \& Quaresma AC (2014) Forófitos preferenciais de orquídeas epífitas na APA Ilha do Combu, Belém, Pará, Brasil. Biota Amazônia 4: 1-4.

Migenis LE \& Ackerman JD (1993) Orchid-phorophyte relationship in a forest watershed in Puerto Rico. Journal of Tropical Ecology 9: 231-240.

Montibeller-Silva K (2018) Orchidaceae em áreas de Floresta Ombrófila Mista: diversidade e ecologia. Tese de Doutorado. Universidade do Estado de Santa Catarina, Lages. 140p.

Nadkarni N \& Solano R (2002) Potential effects of climate change on canopy communities in a tropical cloud forest: an experimental approach. Oecologia 131: 580-586.

Nieder J, Engwald S, Klawun M \& Barthlott W (2000) Spatial distribution of vascular epiphytes (including hemiepiphytes) in a lowland Amazonian rain forest (Surumoni crane plot) of southern Venezuela. Biotropica 32: 385-396.

Oksanen J, Blanchet FG, Friendly M, Kindt R, Legendre P, McGlinn D, Minchin PR, O’Hara RB, Simpson GL, Solymos P, Stevens MHH, Szoecs E \& Wagner H (2018) Package vegan: community ecology package, version 2.4-6. Disponível em $<$ https://cran.r-project.org >; <https://github.com/ vegandevs/vegan $>$. Acesso em 12 fevereiro 2018. 
Otero JT, Aragón S \& Ackerman JD (2007) Site variation in spatial aggregation and phorophyte preference in Psychilis monensis (Orchidaceae). Biotropica 39: 227-231.

Padilha PT, Elias GA, Santos R, Martins R \& Citadini-Zanette V (2017) Vascular epiphytes respond to successional stages and microhabitat variations in a subtropical forest in southern Brazil. Brazilian Journal of Botany 40: 1-9.

Pérez CA, Guevara R, Carmona MR \& Armesto JJ (2005) Nitrogen mineralization in epiphytic soils of an oldgrowth Fitzroya cupressoides forest, southern Chile. Écoscience 12: 210-215.

Prescott C (2002) The influence of the forest canopy on nutrient cycling. Tree Physiology 22: 1193-1200.

Rasmussen HN \& Rasmussen FN (2018) The epiphytic habitat on a living host: reflections on the orchid-tree relationship. Botanical Journal of the Linnean Society 186: 456-472.

Ribeiro JELS, Hopkins MJG, Vicentini A, Sothers CA, Costa MAS, Brito JM, Souza MAD, Martins LHP, Lohmann LG, Assuncão PACL, Pereira EC, Silva CF, Mesquita MR \& Procopio LC (1999) Flora da Reserva Ducke: guia de identificação das plantas vasculares de uma floresta de terra-firme na Amazônia Central. INPADFID, Manaus. 800p.

Ribeiro MC, Metzger JP, Martensen AC, Ponzoni FJ \& Hirota MM (2009) The Brazilian Atlantic Forest: how much is left, and how is the remaining forest distributed? Implications for conservation. Biological Conservation 142: 1141-1153.

Rogalski JM \& Zanin EM (2003) Composição florística de epífitos vasculares no estreito de Augusto César, Floresta Estacional Decidual do Alto Uruguai, RS, Brasil. Revista Brasileira de Botânica 26: 551-556.

Royer CA, Brito ALVT \& Smidt EC (2014) O gênero Phymatidium (Orchidaceae: Oncidiinae) no estado do Paraná. Rodriguésia 65: 251-260.

RStudio Team (2015) RStudio: integrated development for R. Disponível em <http://www.rstudio.com/>. Acesso em 01 fevereiro 2018.

Santos AS, Saraiva DD, Müller SC \& Overbeck GE (2015) Interactive effects of environmental filtering predict beta-diversity patterns in a subtropical forest metacommunity. Perspectives in Plant Ecology, Evolution and Systematics 17: 96-106.

Stadmüller I (1987) Los bosques Nublados em el Trópico Húmedo. Universidad de las Naciones Unidas, San José. $85 \mathrm{p}$.

Tejo CF, Zabowski D \& Nadkarni NM (2015) Total and epiphytic litter under the canopy of Acer macrophyllum in an old-growth temperate rainforest, Washington State, USA. Canadian Journal of Forest Research 45: 1654-1661.
Trapnell DW, Hamrick JL \& Nason JD (2004) Threedimensional fine-scale genetic structure of the neotropical epiphytic orchid, Laelia rubescens. Molecular Ecology 13: 1111-1118.

Triana-Moreno LA, Garzón-Venegas NJ, Sanchez-Zambrano J \& Vargas O (2003) Epífitas vasculares como indicadores de regeneración enbosques intervenidos de la Amazônia Colombiana. Acta Biológica Colombiana 8: $31-42$.

Wagner K, Mendieta-Leiva G \& Zotz G (2015) Host specificity in vascular epiphytes: a review of methodology, empirical evidence and potential mechanisms. AoB Plants 7: 1-25.

Wang X, Long W, Schamp BS, Yang X, Kang Y, Xie Z \& Xiong M (2016) Vascular epiphyte diversity differs with host crown zone and diameter, but not orientation in a tropical Cloud Forest. PLoS ONE 11: e0158548.

Webster GL (1995) The panorama of Neotropical cloud forests. In: Churchill SP (ed.) Biodiversity and conservation of Neotropical montane forests. New York Botanical Garden, New York. Pp. 53-77.

Werner FA \& Gradstein SR (2009) Diversity of dry forest epiphytes along a gradient of human disturbance in the tropical Andes. Journal of Vegetation Science 20: 59-68.

Wolf JHD (1994) Factors controlling the distribution of vascular and nonvascular epiphytes in the Northern Andes. Plant Ecology 112: 15-28.

WolfJHD (2005) The response of epiphytes to anthropogenic disturbance of pine-oak forests in the highlands of Chiapas, Mexico. Forest Ecology and Management 212: 376-393.

Woods CL, Cardelu's CL \& Dewalt SJ (2015) Microhabitat associations of vascular epiphytes in a wet tropical forest canopy. Journal of Ecology 103: 421-430.

Zhang S, Chen W, Huang J, Bi Y \& Yang X (2015) Orchid species richness along elevational and environmental gradients in Yunnan, China. PLoS One 10: 1-23.

Zimmerman JK \& Olmsted IC (1992) Host tree utilization by vascular epiphytes in a seasonally inundated forest (Tintal) in Mexico. Biotropica, 24: 402-407.

Zotz G \& Bader MY (2009) Epiphytic plants in a changing World-Global: change effects on vascular and nonvascular Epiphytes. In: Lüttge U, Beyschlag W, Büdel B \& Francis D (eds.) Progress in Botany. Springer, Berlin. Pp. 147-170.

Zotz G \& Vollrath B (2003) The epiphyte vegetation of the palm Socratea exorrhiza - correlations with tree size, tree age and bryophyte cover. Journal of Tropical Ecology 19: 81-90.

Zotz G (2013) The systematic distribution of vascular epiphytes - a critical update. Botanical Journal of the Linnean Society 171: 453-481. 